\title{
The value of 'gentle reminder' on safe medical behaviour
}

\author{
Ido Erev, ${ }^{1}$ Dotan Rodensky, ${ }^{1}$ Mark-Alain Levi, ${ }^{2}$ Michal Englard-Hershler, ${ }^{2}$ \\ Hanna Admi, ${ }^{2}$ Yoel Donchin ${ }^{3}$
}

${ }^{1}$ The Research Center for Work Safety and Human Engineering, Faculty of Industrial Engineering and Management, Technion, Haifa, Israel

2Mark-Alain Levi, Rambam Medical Center, Haifa, Israel ${ }^{3}$ Yoel Donchin Hadassah Hebrew University Medical Center, Jerusalem, Israel

\section{Correspondence to} Dr Yoel Donchin, Hadassah Hebrew University Medical Center, P0 Box 12007, Jerusalem 91120, Israel; yoeld@ekmd.huji.ac.il

Accepted 18 December 2009 Published Online First 31 May 2010

\section{ABSTRACT}

Background Based on knowledge and methods from cognitive psychology and behavioural economics we introduced 'Gentle reminder.' This procedure calls for public planning of safety norms, and an agreement of all team members to help each other to adhere to this plan. Methods Team members agree to gently remind their coworkers every time they deviate from the safety norm. For the study, we observed the use of gloves during intravenous insertion and blood withdrawal.

Results and discussion During the 2 years of observation, safe behaviour increased, if safe behaviour before implementation of the 'gentle reminder' was 55\% in one ward; it increased after 2 months to above $80 \%$; 2 months later, it was $83 \%$ and $90 \%$; and finally it stabilised on $90 \%$. A similar pattern was documented in all wards. This is one recommended way to overcome unsafe behaviour.

\section{INTRODUCTION}

'To err is human' is the cliche that is being used to explain an accidents and mishaps, not just in medicine. The science of cognitive psychology and the body of knowledge regarding decision-making and rational (or non rational behaviour) were not part of the 'war against error' declared by the Institute of Medicine report. ${ }^{1}$ The path to create a safe medical environment went through reporting of mishaps, better design and leadership. However, 5 years after the Institute of Medicine report, the numbers of medical errors and mishaps are not in the decline. ${ }^{2}$ The introduction of the 'checklist' and the 'time out' procedure before surgery demonstrates that it is possible to reduce the number of mishaps by being proactive and using methods that have a theoretical background from the cognitive psychology. ${ }^{3}{ }^{4}$ We would like to suggest the use of a 'gentle' approach, in which we implement knowledge and methods from cognitive psychology and behavioural economics into the daily routine of a medical ward of a general hospital. By doing this, we could influence and enhance the safety behaviour of the medical team.

\section{BACKGROUND}

Basic research in human decision-making reveals a large gap between decisions that are made based on personal experience and decisions that are made based on a description of the possible outcomes. People tend to underplay rare events (such as 'it won't happen to me') in decisions from experience, ${ }^{4}$ and overplay rare events in decisions from description. ${ }^{5}$ In one of our studies, subjects were to choose between the status quo (payoff of 0 with certainty) and a gamble that promises a gain of 1 Sheqel in $90 \%$ of the trials and a loss of 10 Sheqels in $10 \%$ of the trials. The results show that $60 \%$ of subjects preferred the gamble that impairs their expected earnings when they relied on experience, but only $10 \%$ accepted this gamble when they responded to a description of the payoff distributions. The tendency to underplay rare events in decisions from experience is robust. It is common to humans and lower animals, and can be captured with a simple model that assumes reliance on a small sample of experiences. ${ }^{4} 6$ The tendency to overplay rare events in decisions from description is robust, too, and this pattern explains why people buy lotteries and insurance. ${ }^{5}$

Additional research shows that the experiencedescription gap implies a difference between ongoing decisions and planning. Ongoing decisions tend to be made from experience and reflect underplaying of rare events, and planning decisions are more likely to be made based on description and reflect overplaying of rare events. One example of this gap is the difference between buying and using safety devices and insurance policies. People tend to buy these products, but in many cases they do not use them. For example, many people buy a car radio with a detachable panel but do not detach it. ${ }^{4}$

A direct indication of the gap between planning and ongoing decisions was documented in a study ${ }^{7}$ that focuses on a repeated play (100 trials) of a choice between a safe action, and a risky prospect that saves 0.01 Shekels in $99.5 \%$ of the trials, but leads to a loss of 2 Shekels in $0.05 \%$ of the trials. The participants received a complete description of the incentive structure. In Condition Planning, the participants were asked to plan their action in advance. In Condition Ongoing, the participants were asked to decide before each trail (and they received immediate feedback after each choice). The typical participant preferred the safe alternative in the planning condition, but experience led to a preference of the risky option $(69 \%$ in the last 50 trials)

This 'experience-description gap' has three interesting implications to the design of safe medical environments. First, it implies that it is important to enforce compliance with safety rules. When workers rely on personal experience, they are expected to exhibit insufficient sensitivity to lowprobability risks, even if this behaviour is not beneficial to them.

Second, massive punishments that are provided with low probabilities are not likely to be effective. 
Workers are likely to overplay the risk of these punishments when they plan their actions and buy insurance, but underplay them when they work.

Finally, the experience-description gap suggests that safety can be enhanced by relying on the fact that workers plan to behave safely.

The current paper focuses on one implementation of this idea that implies a 'gentle reminder' procedure. This procedure calls for public planning of safety norms, and an agreement of all team members to help each other to adhere to this plan. That is, team members agree to gently remind their coworkers every time that they deviate from the safety norm.

The potential of this 'gentle reminder' procedure was documented in studies that address cheating in exams, and enhancing safety in five industrial plants in Israel. ${ }^{8}$ This research suggests that gentle reminders are expected to be effective under two conditions. First, the probability of receiving gentle reminders when violating the norm should be high. Second, the experience of receiving a gentle reminder is less pleasant than the experience of behaving safely.

The current logic implies that under these conditions, using soft remarks like 'you forget to wear gloves' is a better way of intervening and does not cause negative feelings. Our hypothesis was that we shall be able to change the behaviour of medical teams regarding a very basic action, namely adherence to sterile procedure during blood sample withdrawal and insertion of intravenous lines.

\section{STUDY DESIGN}

The study took place at the Rambam Medical Center, which is a tertiary care hospital of 900 beds in Haifa, Israel. Five internal medicine departments participated in the study. In the preintervention study, observers (who looked like typical visitors) were asked to record whether the physicians and nurses are adhering to the protocol regarding the use of gloves before and after intravenous insertion and blood withdrawal. One hundred per cent safety behaviour means that the physician or nurse follows the instructions as written, and deviation from parts of the protocol yielded a lower score of safety. Before the second stage, we present the 'gentle reminders' idea, as described above; the five teams agreed that this idea is worth trying. All agreed that they 'sometimes fail' to use gloves for the procedures, would like their coworkers to remind them to do so and want to help their coworkers to do the same.

In order to evaluate the effect of this minimalistic intervention, we continued the observation procedure, described above. At the first stage of the study (the first 12 months), the observations took place at least four times a week, during all shifts (with the exception of 2 months during the Second Lebanon war), and the frequency of observations was reduced to once a month later. The outcomes of the observations were presented to the department head once a month.

\section{RESULTS}

Figure 1 (physicians) shows how, during the 2 years of observation, safe behaviour increased, if safe behaviour before implementation of the 'gentle enforcement' was $55 \%$ in one ward, and increased after 2 months to above $80 \%$ and 2 months later to $83 \%$ and $90 \%$, finally stabilising at $90 \%$. Very similar results were achieved in all other wards. As a matter of fact, the hospital authority started to investigate why there was such an increase in the use of sterile gloves. One exception is the slow start in ward 4. This pattern appears to be a result of the initial attitude of the head nurse. She believes that the cost of using

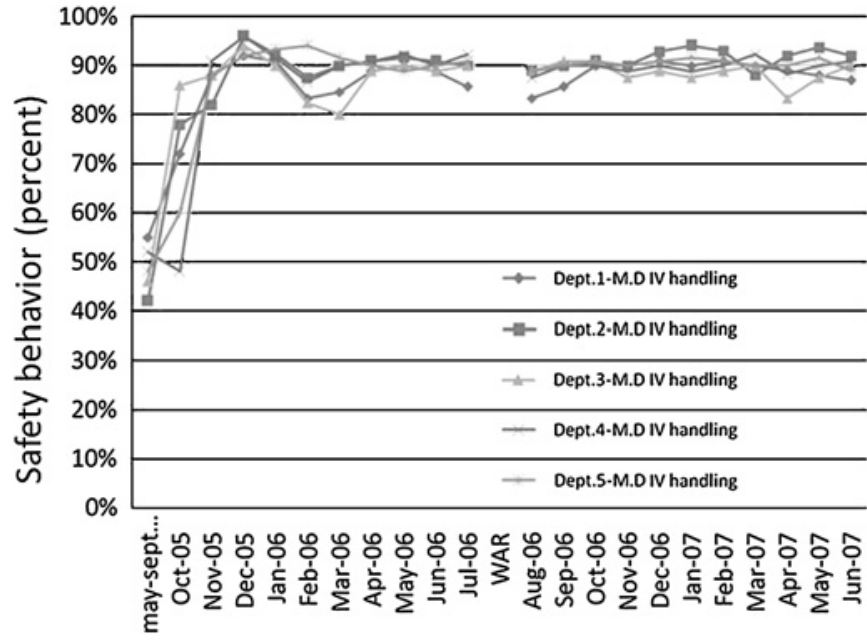

Figure 1 The percentage of safe behaviours by the physicians as a function of time in the five departments. The results for the nurses were similar with a slower increase in Department 4 . The first observation presents the pre-intervention baseline.

gloves is larger than the benefit. Only after 6 months did she decide to follow the new norm.

Interviews suggest that the number of explicit reminders per ward in the first month was around five a day. The most common reminders $(50 \%)$ were from nurses to physicians. The frequency of explicit reminders decreased with time. The relative low frequency of reminders suggests that the larger effect is not an immediate response to specific reminders; rather, it is more likely to be an attempt to avoid reminders.

\section{DISCUSSION}

It is constructive to distinguish between three sources of deviations from safe medical procedures: situations in which workers 'do not want,' 'cannot' or 'want, can but do not' (wacabudo-no) behave safely. Most previous analyses focus on the first two sources, the current analysis focuses on the third. It highlights two interesting observations. First, it shows that this source of deviations can be more important than suggested by traditional analyses. Recent research shows a robust and consistent description-experience gap. In many cases, people plan to behave safely but deviate from their plan. Second, it is easy to address the 'want, can but do not' problems. They can be eliminated with the gentle reminder procedure.

The study described above highlights the significance of these observations. It shows that in many cases (about $45 \%$ of our baseline observations), medical personal can, but do not, use gloves during intravenous handling. This problematic behaviour appears to be the norms in certain settings. Nevertheless, a surprisingly simple and cheap intervention, the implementation of the 'gentle reminder' procedure, dramatically changed this behaviour. The observation did not influence the behaviour and in the 2 months during the war, where there were no observations at all, the behaviour stayed the same.

Safety is a value, not a commodity. It is not enough to add computers or sophisticated barcode reading in order to reduce the error rate. The way to achieve safety is by changing the behaviour of the human operator, as this is being called by human factors engineers. The current analysis shows that in certain cases, the 'gentle intervention' is sufficient to trigger a significant change.

Competing interests None. 
Ethics approval Ethics approval was provided by Rambam Medical Center.

Provenance and peer review Not commissioned; externally peer reviewed.

\section{REFERENCES}

1. To err is human. Building a safer health system. Committee on quality of health care in America. Institute of Medicine report. 2000.

2. Leape LL, Berwick DM. Five years after to err is human: what have we learned? JAMA 2005:293:2384-90.

3. Barron G, Erev I. Small feedback-based decisions and their limited correspondence to description based decisions. J Behav Decis Mak 2003;16:215-33.
4. Erev I, Barron G. On adaptation, maximization, and reinforcement learning among cognitive strategies. Psychol Rev 2005;112:912-31.

5. Kahneman D, Tversky A. Prospect theory: an analysis of decision under risk. Econometrica 1979:47:263-91.

6. Shafir S, Reich T, Tsur E, et al. Perceptual accuracy and conflicting effects of certainty on risk-taking behaviour. Nature 2008;453:917-21.

7. Yechiam E, Barron G, Erev I. Description, experience and the effect of rare terrorist attacks. J Conflict Resolut 2005:49:430-9.

8. Erev I. On the weighting of rare events and the economics of small decisions. In: Oda $\mathrm{SH}$ ed. Advances in experimental economics, lecture notes in economics and mathematical systems. Dordrecht, The Netherlands: Springer, 2007:590-7. 